functional-connectivity between these areas by presenting words classified along the attributes of frequency, concreteness, and regularity, which utilize specific cognitive routes, and a visual control. Connectivity is analyzed during word reading overall vs. a control condition to determine overall reading-related connectivity, and while reading words that have high vs. low attribute values, to determine if cognitive processing routes bias the neural reading network connectivity. RESULTS/ANTICIPATED RESULTS: The localizer analysis is expected to result in the activation of canonical reading areas. The degree of functional connectivity observed between these regions is expected to depend on the degree to which each cognitive route is utilized to read a given word. After orthographic, phonologic, and semantic areas have been identified, the connectivity analysis should show that there is high correlation between all three types of areas during reading compared to the control condition. Then the frequency, regularity, and concreteness of the words being read should alter the reliance on the pathways between these area types. This would support the hypothesized pattern of connectivity as predicted by the cognitive reading routes. Otherwise, it will show how the neural reading network differs from the cognitive model. DISCUSSION/SIGNIFICANCE OF IMPACT: The results will determine the relationship between the cognitive reading model and the neural reading network. Cognitive models show what processes occur in the brain, but neural networks show how these processes occur. By relating these components, we obtain a more complete view of reading in the brain, which can inform future alexia treatments.

4341

Neuroclinical fingerprints of risk for psychosis: Profiles of neurophysiology, symptom severity, and cognitive function

Keisha Novak ${ }^{1}$, Sam Buck ${ }^{1}$, Roman Kotov ${ }^{2}$, and Dan Foti ${ }^{1}$

${ }^{1}$ Purdue University; ${ }^{2}$ Stony Brook University

OBJECTIVES/GOALS: The study aims to utilize event-related potentials (ERPs) coupled with observable reports of symptoms to comprehensively understand neurological and symptomatic profile of individuals at risk for developing psychosis. The study is a shortterm longitudinal design which allows for examination of course as well as structure of illness. METHODS/STUDY POPULATION: This study uses a combination of well-validated ERPs (P300, $\mathrm{N} 400, \mathrm{ERN}$ ) and symptom data to predict variation in symptoms over time. We parse heterogeneity within a high-risk group to create innovative profiles and predict variation in course of symptoms. Data collection is ongoing $(n=35$; target $N=100)$. Methods include a battery of ERP tasks tracking neural processes associated with attention, language processing, and executive function (P300, N400, ERN), along with assessment of symptom type and severity. Analyses include how ERPs correlate with severity of risk and symptom dimensions (positive, negative, disorganized). We examine whether individual versus global ERP aberrations (P300, N400, ERN) predict individual versus global symptom domain severity (positive, negative, disorganized), or vice versa. RESULTS/ ANTICIPATED RESULTS: Symptom domain scores were elevated compared to general population on positive $(M=1.65, S D=.36)$, negative $(M=1.9 S D=.42)$, and depressive $(M=1.94, S D=.40)$ domains. Small to medium effect sizes emerged for P300 profile ( $r$ 's $=-.001$ to -.41 ) and ERN profile (r's $=-.03$ to -.37 ), though small effect sizes for N400 profile ( $r$ 's $=-.06$ to .29 ). Analyses were run to determine the degree to which profiles of risk were similar:
P300/ERN $\quad(r=-.09), \quad$ ERN/N400 $\quad(r=-.39), \quad$ and $\quad \mathrm{N} 400 / \mathrm{P} 3$ $(\mathrm{r}=-.20)$. Additional analyses suggest potential mediating effects of cognition on neural activity and symptoms. DISCUSSION/ SIGNIFICANCE OF IMPACT: We use a combination of well-validated ERPs (i.e. P300, N400, ERN) with behavioral and symptom data to predict variation in symptoms over time. A "fingerprint" physiologic aberration may be exhibited within high-risk individuals and can be used as biomarkers to identify those at risk even before onset of observable symptoms.

4532

Pancreatic Cyst Risk Stratification for Early Detection of Pancreatic Cancer Using Quantitative Radiomics and Activity-Based Biomarkers

Sophia Hernandez ${ }^{1}$, Andre Luiz Lourenco, Evan Calabrese, Tyler

York, Alexa Glencer, Spencer Behr, Zhen Jane Wang, Eugene Koay, Charles Craik, and Kimberly Kirkwood

${ }^{1}$ University Of California, San Francisco

OBJECTIVES/GOALS: Pancreatic cysts are comprised of both precancerous mucinous lesions and non-mucinous lesions with minimal malignant potential. Our goal is to improve our ability to classify the type of cyst using a combination of novel radiomic features and cyst fluid proteolytic activity. METHODS/STUDY POPULATION: Preoperative pancreatic protocol CT images from 30 patients with proteolytic assay characterization, followed by surgical resection with a pathologically confirmed pancreatic cyst diagnosis between 20162019 will be used in this study. We will contour images using the widely available software 3D Slicer, and extract radiomic features using IBEX software. We will analyze area under the ROC curves to identify the radiomic features that best differentiate mucinous from non-mucinous cysts, and identify features to be cross validated. The predictive ability of identified radiomic features combined with proteolytic assay will be determined by performing multiple logistic regression analysis and comparing AUROC analysis. We will determine sensitivity and specificity for individual, as well as combinations of, analytes to determine the optimal classifier. RESULTS/ANTICIPATED RESULTS: We anticipate that the predictive ability, sensitivity, and specificity of utilizing radiomic features combined with proteolytic assay data will exceed the performance of any individual test. DISCUSSION/ SIGNIFICANCE OF IMPACT: This work is designed to provide a predictive radiomic model that will enable us to better identify mucinous cysts that require further evaluation, and potentially prevent unnecessary surgery in other patients. Ultimately, we would like to improve the accuracy of noninvasive radiographic evaluation using radiomic markers. CONFLICT OF INTEREST DESCRIPTION: Dr. Charles Craik is a co-founder of Alaunus Biosciences, Inc.

4340

\section{Piloting Implementation and Dissemination of Best Practice Guidelines Using BPM+Health}

James McClay, MD, MS, FACEP, FAMIA ${ }^{1}$, and Pawan Goyal ${ }^{2}$

${ }^{1}$ University of Nebraska Medical Center - Great Plains IDeA-CTR;

${ }^{2}$ American College of Emergency Physicians

OBJECTIVES/GOALS: Clinical translational studies inform clinical practice patterns through dissemination of clinical practice guidelines (CPG). In EM practices change to rapidly for timely local EHR implementation. We test the OMG BPM+Health specification for rapid deployment of best practices relevant to EM. METHODS/STUDY 